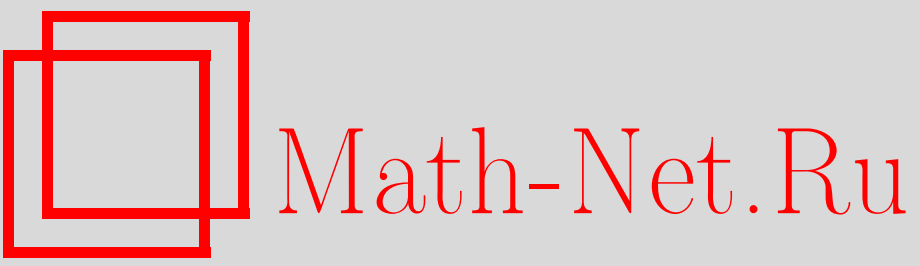

А. В. Антонов, Квантовая модель Вольтерра и универсальная $R$-матрица, $T M \Phi$, 1997, том 113, номер 3, 384-396

DOI: https://doi.org/10.4213/tmf1087

Использование Общероссийского математического портала Math-Net.Ru подразумевает, что вы прочитали и согласны с пользовательским соглашением

http://www.mathnet.ru/rus/agreement

Параметры загрузки:

IP : 54.237 .59 .107

26 апреля 2023 г., 15:56:42 
ТЕОРЕТИЧЕСКАЯ

И МАТЕМАТИЧЕСКАЯ

ФИЗИКА

Том 113, № 3

декабрь, 1997

А.В. Антонов*

\section{КВАНТОВАЯ МОДЕЛЬ ВОЛЬТЕРРА И УНИВЕРСАЛЬНАЯ $R$-МАТРИЦА}

Показано, что интегрируемая система, решаемая методом обратной задачи рассеяния, может быть описана с помощью чисто алгебраического объекта (универсальной $R$-матрицы) и некоторого представления. Именно, на примере квантовой модели Вольтерра мы выписываем $L$-оператор и фундаментальную $R$-матрицу исходя из универсальной $R$-матрицы для квантовой аффинной алгебры $U_{q}\left(\widehat{s l}_{2}\right)$ и представления $q$-осцилляторов для нее. Таким образом, существует эквивалентность между интегрируемой системой с алгеброй симметрий $\mathcal{A}$ и представлением этой алгебры.

\section{ВВЕДЕНИЕ}

Напомним, что классическая модель Вольтерра [1] является разностно-дифференшиальным уравнением

$$
\frac{d s_{n}}{d t}=s_{n}\left(s_{n+1}-s_{n-1}\right) .
$$

Индекс $n$ принадлежит множеству целых чисел. Модель Вольтерра описывает популяционную динамику, а также скорость некоторых химических реакций. Это уравнение имеет приложения и в физике [1]. Кроме того, оно является решеточным аналогом модели Лиувилля [2]. Интегрируемость модели Вольтерра была показана в [1].

Гамильтонова формулировка для периодических граничных условий $s_{n+N}=s_{n}$ следуюшая [2]:

$$
\frac{d s_{n}}{d t}=\left\{H, s_{n}\right\}
$$

гамильтониан

$$
H=-\frac{1}{\gamma} \sum_{n=1}^{N} \ln s_{n},
$$

скобки Пуассона

$$
\left\{s_{m}, s_{n}\right\}=-\frac{1}{2} \gamma\left[\left(4-s_{m}-s_{n}\right)\left(\delta_{m+1, n}-\delta_{m-1, n}\right)+s_{m-1} \delta_{m-2, n}-s_{m+1} \delta_{m+2, n}\right],
$$

здесь $\gamma$ - константа связи.

${ }^{*}$ Институт теоретической физики им. Л. Д. Ландау, Москва, Россия; Laboratoire de Physique Théorique et Hautes Energies Université Pierre et Marie Curie, Paris, France. E-mail: antonov@lpthe.jussieu.fr., antonov@landau.ac.ru. 
Перейдем к квантовому случаю модели Вольтерра $[3,4]$. Введем на $\mathbb{Z}$ квантовые поля $v_{\alpha}, u_{\beta}$, удовлетворяющие условию

$$
u_{i} v_{i}=q v_{i} u_{i}
$$

причем поля в различных точках цепочки коммутируют. Чтобы построить квантовый гамильтониан, зададим переменные

$$
w_{\alpha \beta}=u_{\alpha}^{-1} u_{\beta}^{-1} v_{\alpha}^{-1} v_{\beta}
$$

с коммутационными соотношениями

$$
w_{n+1, n} w_{n, n-1}=q^{2} w_{n, n-1} w_{n+1, n}
$$

Для примера рассмотрим случай $q^{2 M+1}=1$ и положим $u^{2 M+1}=v^{2 M+1}=1$. Тогда квантовый гамильтониан Вольтерра [3] является полиномом по $w^{ \pm 1}$ :

$$
H=\sum_{n=1}^{N} H\left(w_{n+1, n}\right),
$$

где $H(w)=h(w)+h\left(w^{-1}\right)$ и

$$
h(w)=\sum_{j=1}^{M} \frac{(-1)^{j}}{q^{j}-q^{-j}} w^{j} .
$$

В общем случае гамильтониан содержит бесконечные ряды по $w^{ \pm 1}$.

Квантовый $L$-оператор модели Вольтерра [3] в точке $\alpha$

$$
L_{\alpha}(\lambda)=\left(\begin{array}{cc}
u_{\alpha} & -\lambda v_{\alpha}^{-1} \\
\lambda v_{\alpha} & u_{\alpha}^{-1}
\end{array}\right)
$$

удовлетворяет уравнению Янга-Бакстера (ЯБ) для тригонометрической $4 \times 4 R$-матрицы. Используя квантовый метод обратной задачи рассеяния (KMO3) [5], можно получить квантовый гамильтониан Вольтерра (4) из $L$-оператора (5).

Возьмем классический предел квантовой модели и установим связь между переменными $w_{\alpha \beta}^{(\mathrm{cl})}$ и $s_{n}$. В пределе коммутационные соотношения (3) переходят в соотношения

$$
\left\{w_{n+1, n}^{(\mathrm{cl})}, w_{n, n-1}^{(\mathrm{cl})}\right\}=-2 w_{n, n-1}^{(\mathrm{cl})} w_{n+1, n}^{(\mathrm{cl})} .
$$

Легко видеть, что выражение (так называемое преобразование Миуры на решетке [2])

$$
s_{n}=\frac{1}{\left(1+w_{n+1, n}^{(\mathrm{cl})}\right)\left(1+\left(w_{n+1, n}^{(\mathrm{cl})}\right)^{-1}\right)}
$$

имеет скобки Пуассона (2). Квантовьй гамильтониан (4) в пределе $q \rightarrow 1, M \rightarrow \infty$ переходит в классический (1).

2 Теоретическая и математическая физика, т. 113, № 3, 1997 г. 
Как было отмечено выше, КМОЗ дает набор квантовых интегралов движения из квантового $L$-оператора. Производяшая функция для интегралов движения есть след матрицы монодромии, зависяшей от спектрального параметра. Для периодических граничных условий матрица монодромии имеет вид $M(\lambda)=L_{N}(\lambda) \ldots L_{2}(\lambda) L_{1}(\lambda)$.

Другой подход к объектам КМОЗ основывается на так называемой универсальной $R$-матрице. Явная формула для нее в случае квантовых аффинных алгебр была найдена в $[6]$.

Для некоторой алгебры $\mathcal{A}$ универсальная $R$-матрица лежит в квадрате $R \in \mathcal{A} \otimes \mathcal{A}$ и удовлетворяет уравнению ЯБ

$$
R_{12} R_{13} R_{23}=R_{23} R_{13} R_{12}
$$

в $\mathcal{A} \otimes \mathcal{A} \otimes \mathcal{A}$. Мы пишем $R=\sum_{i} A_{i} \otimes B_{i} \in \mathcal{A} \otimes \mathcal{A}$ и полагаем

$$
R_{12}=\sum_{i} A_{i} \otimes B_{i} \otimes 1, \quad R_{13}=\sum_{i} A_{i} \otimes 1 \otimes B_{i}, \quad R_{23}=\sum_{i} 1 \otimes A_{i} \otimes B_{i}
$$

Интегрируемая система с симметрией $\mathcal{A}$, решаемая КМОЗ, может быть описана с помощью универсальной $R$-матрицы $R \in \mathcal{A} \otimes \mathcal{A}$ для алгебры $\mathcal{A}$ и ее некоторого представления. Действительно, представление первой алгебры $\mathcal{A}$ из $R$ (квантовое представление) связано с рассматриваемой интегрируемой системой, в то время как представление второй алгебры из $R$ (вспомогательное представление) определяет тип объекта КМОЗ ( $L$-оператор, фундаментальная $R$-матрица, $Q$-оператор Бакстера и т.д.) [7].

Ниже мы рассмотрим случай алгебры $U_{q}\left(\widehat{s l}_{2}\right)$. Матрица монодромии может быть получена из универсальной $R$-матрицы $R \in U_{q}\left(\widehat{s l}_{2}\right) \otimes U_{q}\left(\widehat{s l}_{2}\right)$ с помощью некоторого квантового представления (зависяшего от рассматриваемой интегрируемой системы) для первой алгебры $U_{q}\left(\widehat{s l}_{2}\right)$ и вспомогательного матричного $(n \times n)$-представления со спектральным параметром для второй алгебры $U_{q}\left(\widehat{s l}_{2}\right)$ в квадрате $U_{q}\left(\widehat{s l}_{2}\right) \otimes U_{q}\left(\widehat{s l}_{2}\right)$.

Другим примером является $Q$-оператор Бакстера $Q(\lambda)$, удовлетворяющий уравнению Бакстера [8]

$$
T(\lambda) Q(\lambda)=Q\left(q^{2} \lambda\right)+Q\left(q^{-2} \lambda\right)
$$

Мы можем получить оператор Бакстера из универсальной $R$-матрицы $R \in U_{q}\left(\widehat{s l}_{2}\right) \otimes$ $U_{q}\left(\widehat{s l}_{2}\right)$, используя некоторое квантовое представление для первой алгебры $U_{q}\left(\widehat{s l}_{2}\right)$ и вспомогательное бесконечномерное представление $q$-осцилляторов $[9,10]$ для второй алгебры $U_{q}\left(\widehat{s l}_{2}\right)$ в квадрате $U_{q}\left(\widehat{s l}_{2}\right) \otimes U_{q}\left(\widehat{s l}_{2}\right)$.

Согласно методам получения матрицы монодромии или $Q$-оператора из универсальной $R$-матришы мы определяем только представление второй алгебры $U_{q}\left(\widehat{s l}_{2}\right)$ в $R \in$ $U_{q}\left(\widehat{s l}_{2}\right) \otimes U_{q}\left(\widehat{s l}_{2}\right)$, в то время как представление первой алгебры, определяющее конкретную рассматриваемую модель, не фиксировано. Таким образом, применение универсальной $R$-матришы дает универсальную технику для вывода алгебраических соотношений между различными объектами КМОЗ [11].

В этой статье рассматривается бесконечномерноепредставление $q$-осцилляторов как квантовое для универсальной $R$-матрицы. Оказывается, что интегрируемая модель, описываемая таким представлением, является квантовой моделью Вольтерра [3, 4]. Мы 
выводим все объекты КМОЗ для системы Вольтерра, такие как $L$-оператор и фундаментальная $R$-матрица [3], из универсальной $R$-матрицы, используя представление $q$-осцилляторов как квантовое.

В разделе 1 мы напоминаем некоторые ключевые моменты КМОЗ и интерпретируем матрицу монодромии как представление универсальной $R$-матрицы. В разделе 2 приведена явная конструкция $L$-оператора модели Вольтерра из универсальной $R$-матрицы. В разделе 3 мы выводим фундаментальную $R$-матрицу модели Вольтерра из универсальной $R$-матрицы. Раздел 4 содержит обсуждение полученных результатов.

Автор признателен Л. Болье и Laboratoire de Physique Théorique et Hautes Énergies (Париж, Франция) за гостеприимство. Автор благодарен А. А. Белавину, А. Ю. Волкову, Ф.А. Смирнову, Л. Д. Фаддееву, Б. Л. Фейгину, С.М. Хорошкину и Б. Энрикесу за полезные обсуждения, а также М. Кулон за поддержку.

\section{1. КВАНТОВЫЙ МЕТОД ОБРАТНОЙ ЗАДАЧИ РАССЕЯНИЯ И УНИВЕРСАЛЬНАЯ $R$-МАТРИЦА}

В этом разделе мы используем обозначения работы [7]. Квантовый $L$-оператор является основным объектом KMO3. $L_{n, a}(\lambda)$ есть матрица во вспомогательном пространстве $\nu_{a}$, в то время как матричные элементы $L_{n, a}(\lambda)$ - операторы в гильбертовом пространстве $h_{n}, n=1, \ldots, N$, связанном с точкой на решетке, $\lambda$ - спектральный параметр. Операторы в различных точках решетки коммутируют. Таким образом, $L_{n, a}(\lambda)$ - оператор в $h_{n} \otimes \nu_{a}$.

Коммутационные соотношения для матричных элементов $L_{n, a}(\lambda)$ записываются в виде уравнения ЯБ

$$
L_{n, a_{1}}(\mu) L_{n, a_{2}}(\lambda) R_{a_{1}, a_{2}}(\lambda / \mu)=R_{a_{1}, a_{2}}(\lambda / \mu) L_{n, a_{2}}(\lambda) L_{n, a_{1}}(\mu) .
$$

Это уравнение в $h_{n} \otimes \nu_{a_{1}} \otimes \nu_{a_{2}}$. Индексы $a_{1}, a_{2}$ и переменные $\lambda$ и $\mu$ связаны со вспомогательными пространствами $\nu_{1}$ и $\nu_{2}$, соответственно, $R_{a_{1}, a_{2}}$ - матрица в пространстве $\nu_{a_{1}} \otimes \nu_{a_{2}}$.

Ниже мы будем рассматривать только специальную форму $R_{a_{1}, a_{2}}$-матрицы, так называемую тригонометрическую $R$-матрищу $4 \times 4$. Многие интересные модели на решетке ( $X X Z$-спиновая модель, решеточное уравнение синус-Гордон, система Вольтерра и т.д.) описываются тригонометрической $R$-матрицей.

Другой важный объект КМОЗ - матрица монодромии

$$
M_{a}(\lambda)=L_{N, a}(\lambda) L_{N-1, a}(\lambda) \ldots L_{1, a}(\lambda)
$$

Матрица монодромии удовлетворяет уравнению ЯБ (7). Из этого следует, что следы матриц монодромии $T_{a}(\lambda)=\operatorname{tr}_{\nu_{a}} M_{a}(\lambda)$ с разными спектральными параметрами по вспомогательным пространствам коммутируют, т.е.

$$
\left[T_{a_{1}}(\lambda), T_{a_{2}}(\mu)\right]=0
$$

Таким образом, след матрицы монодромии является производящей функцией для интегралов движения. 
Полезно рассмотреть так называемую фундаментальную $R$-матрицу [12] $R_{n_{1}, n_{2}}(\lambda)$, т.е. оператор в $h_{n_{1}} \otimes h_{n_{2}}$ (вспомогательное и квантовое пространства совпадают). След матрицы монодромии, построенный по фундаментальной $R$-матрице, дает набор локальных интегралов движения.

С другой стороны, возможно описать все объекты КМОЗ (тригонометрическую $R$-матрицу, $L$-оператор и матрицу монодромии, а также фундаментальную $R$-матрицу) с помощью одной алгебраической структуры - универсальной $R$-матрицы. Напомним некоторые факты о ней [6].

Рассмотрим простейший случай аффинной квантовой $U_{q}\left(\widehat{s l}_{2}\right)$-алгебры с матрицей Картана

$$
A=\left(a_{i j}\right), \quad i, j=0,1 ; \quad A=\left(\begin{array}{cc}
2 & -2 \\
-2 & 2
\end{array}\right) \text {. }
$$

Это ассоциативная алгебра с генераторами $e_{ \pm \alpha_{i}}, k_{\alpha_{i}}^{ \pm 1}$ (равными $\left.q^{ \pm h_{\alpha_{i}}}\right), i=0,1$, и коммутационными соотношениями

$$
\begin{gathered}
{\left[k_{\alpha_{i}}^{ \pm 1}, k_{\alpha_{j}}^{ \pm 1}\right]=0, \quad k_{\alpha_{i}} e_{ \pm \alpha_{j}}=q^{ \pm\left(\alpha_{i}, \alpha_{j}\right)} e_{ \pm \alpha_{j}} k_{\alpha_{i}},} \\
{\left[e_{\alpha_{i}}, e_{-\alpha_{j}}\right]=\delta_{i j} \frac{k_{\alpha_{i}}-k_{\alpha_{i}}^{-1}}{q-q^{-1}},} \\
\left(\operatorname{ad}_{q^{\prime}} e_{ \pm \alpha_{i}}\right)^{1-a_{i j}} e_{ \pm \alpha_{j}}=0 \quad \text { для } \quad i \neq j, \quad q^{\prime}=q, q^{-1},
\end{gathered}
$$

где $\left(\operatorname{ad}_{q} e_{\alpha}\right) e_{\beta}-q$-коммутатор:

$$
\left(\operatorname{ad}_{q} e_{\alpha}\right) e_{\beta} \equiv\left[e_{\alpha}, e_{\beta}\right]_{q}=e_{\alpha} e_{\beta}-q^{(\alpha, \beta)} e_{\beta} e_{\alpha}
$$

a $(\alpha, \beta)$ - скалярное произведение корней $\alpha$ и $\beta:\left(\alpha_{i}, \alpha_{j}\right)=a_{i j}$. Наложено также условие $k_{\alpha_{0}} k_{\alpha_{1}}=1$.

Определим “ко-умножение" в алгебре $U_{q}\left(\widehat{s l}_{2}\right)$ :

$$
\begin{gathered}
\Delta\left(k_{\alpha_{i}}\right)=k_{\alpha_{i}} \otimes k_{\alpha_{i}}, \\
\Delta\left(e_{\alpha_{i}}\right)=e_{\alpha_{i}} \otimes k_{\alpha_{i}}+1 \otimes e_{\alpha_{i}}, \quad \Delta\left(e_{-\alpha_{i}}\right)=e_{-\alpha_{i}} \otimes 1+k_{\alpha_{i}}^{-1} \otimes e_{-\alpha_{i}},
\end{gathered}
$$

и “скрученное ко-умножение"

$$
\begin{gathered}
\Delta^{\prime}\left(k_{\alpha_{i}}\right)=k_{\alpha_{i}} \otimes k_{\alpha_{i}} \\
\Delta^{\prime}\left(e_{\alpha_{i}}\right)=k_{\alpha_{i}} \otimes e_{\alpha_{i}}+e_{\alpha_{i}} \otimes 1, \quad \Delta^{\prime}\left(e_{-\alpha_{i}}\right)=1 \otimes e_{-\alpha_{i}}+e_{-\alpha_{i}} \otimes k_{\alpha_{i}}^{-1} .
\end{gathered}
$$

По определению универсальная $R$-матрица - объект в $U_{q}\left(\widehat{s l}_{2}\right) \otimes U_{q}\left(\widehat{s l}_{2}\right)$ такой, что $\Delta^{\prime}(g) R=R \Delta(g)$ для любого $g \in U_{q}\left(\widehat{s l}_{2}\right)$ и

$$
R_{12} R_{13} R_{23}=R_{23} R_{13} R_{12}
$$


Это уравнение является универсальной формой уравнения ЯБ в $U_{q}\left(\widehat{s l}_{2}\right) \otimes U_{q}\left(\widehat{s l}_{2}\right) \otimes$ $U_{q}\left(\widehat{s l}_{2}\right)$. Как и во введении, мы пишем $R=\sum_{i} A_{i} \otimes B_{i} \in U_{q}\left(\widehat{s l}_{2}\right) \otimes U_{q}\left(\widehat{s l}_{2}\right)$ и полагаем

$$
R_{12}=\sum_{i} A_{i} \otimes B_{i} \otimes 1, \quad R_{13}=\sum_{i} A_{i} \otimes 1 \otimes B_{i}, \quad R_{23}=\sum_{i} 1 \otimes A_{i} \otimes B_{i}
$$

Наряду с коммутационными соотношениями для алгебры $U_{q}\left(\widehat{s l_{2}}\right)$ нам понадобится также антиинволюция $*$, определенная как $\left(k_{\alpha_{i}}\right)^{*}=k_{\alpha_{i}}^{-1},\left(e_{ \pm \alpha_{i}}\right)^{*}=e_{\mp \alpha_{i}},(q)^{*}=q^{-1}$.

Мы также пользуемся стандартными обозначениями для $q$-экспонент и $q$-чисел

$$
\exp _{q}(x)=1+x+\frac{x^{2}}{(2)_{q} !}+\cdots+\frac{x^{n}}{(n)_{q} !}+\cdots=\sum_{n \geq 0} \frac{x^{n}}{(n)_{q} !}
$$

где

$$
(a)_{q}=\frac{q^{a}-1}{q-1}, \quad[a]_{q}=\frac{q^{a}-q^{-a}}{q-q^{-1}} .
$$

Определим теперь генераторы Картана-Вейля алгебры $U_{q}\left(\widehat{s l_{2}}\right)$. Пусть $\alpha_{1}$ и $\alpha_{0}=$ $\delta-\alpha_{1}$ суть простые корни аффинной алгебры $\widehat{s l}_{2}$. Тогда $\delta=\alpha_{1}+\beta$ есть минимальный мнимый корень. Зафиксируем следующее нормальное упорядочение системы положительных корней:

$$
\alpha_{1}, \alpha_{1}+\delta, \alpha_{1}+2 \delta, \ldots, \delta, 2 \delta, \ldots, \alpha_{0}+2 \delta, \alpha_{0}+\delta, \alpha_{0}
$$

Положим

$$
\begin{aligned}
e_{\delta}^{\prime} & =e_{\delta}=\left[e_{\alpha_{1}}, e_{\alpha_{0}}\right]_{q}, \\
e_{\alpha_{1}+l \delta} & =(-1)^{l}\left([2]_{q}\right)^{-l}\left(\operatorname{ad} e^{\prime}\right)^{l} e_{\alpha_{1}}, \\
e_{\alpha_{0}+l \delta} & =\left([2]_{q}\right)^{-l}\left(\operatorname{ad} e^{\prime}\right)^{l} e_{\alpha_{0}}, \\
e^{\prime}{ }_{l \delta} & =\left[e_{\alpha_{1}+(l-1) \delta}, e_{\alpha_{0}}\right]_{q} .
\end{aligned}
$$

Обозначим через $E(z)$ и $E^{\prime}(z)$ производящие функции для $e_{n \delta}$ и $e_{n \delta}^{\prime}$ :

$$
E(z)=\sum_{n \geq 1} e_{n \delta} z^{-n}, \quad E^{\prime}(z)=\sum_{n \geq 1} e^{\prime}{ }_{n \delta} z^{-n},
$$

связанные соотношением

$$
\left(q-q^{-1}\right) E(z)=\log \left(1+\left(q-q^{-1}\right) E^{\prime}(z)\right) .
$$

Векторы, соответствующие отрицательным корням, имеют вид $e_{-\gamma}=e_{\gamma}^{*}$.

Универсальная $R$-матрица для $U_{q}\left(\widehat{s l}_{2}\right)$ выглядит следуюшим образом [6]:

$$
\begin{aligned}
\mathcal{R}= & \left(\prod_{n \geq 0}^{\rightarrow} \exp _{q^{-2}}\left(\left(q-q^{-1}\right) e_{\alpha_{1}+n \delta} \otimes e_{-\alpha_{1}-n \delta}\right)\right) \times \\
& \times \exp \left(\sum_{n>0}\left(q-q^{-1}\right) \frac{n\left(e_{n \delta} \otimes e_{-n \delta}\right)}{[2 n]_{q}}\right) \times \\
& \times\left(\prod_{n \geq 0}^{\leftarrow} \exp _{q^{-2}}\left(\left(q-q^{-1}\right) e_{\alpha_{0}+n \delta} \otimes e_{-\alpha_{0}-n \delta}\right)\right) \times \mathcal{K}
\end{aligned}
$$


где порядок перемножения по $n$ прямой в первом произведении и обратный по втором. Множитель $\mathcal{K}$ определяется формулой

$$
\mathcal{K}=q^{\frac{h_{\alpha} \otimes h_{\alpha}}{2}}
$$

Мы видим из (12), что универсальная $R$-матрица принадлежит $U_{q}\left(b_{+}\right) \otimes U_{q}\left(b_{-}\right)$, где $b_{ \pm}$- положительная (отрицательная) борелевская подалгебра алгебры $U_{q}\left(\widehat{s l}_{2}\right)$, образованная $e_{\alpha_{i}}, k_{\alpha_{i}}^{ \pm 1}$ и $e_{-\alpha_{i}}, k_{\alpha_{i}}^{ \pm 1}(i=0,1)$, соответственно.

Можно получить объекты КМОЗ из универсальной $R$-матрицы, используя конкретные представления алгебры $U_{q}\left(\widehat{s l}_{2}\right)$. Например, тригонометрическая $4 \times 4 R$-матрица получается из универсальной с помощью матричного $(2 \times 2)$-представления со спектральным параметром.

Можно получить оператор $L_{n, a}(\lambda)$ из универсальной $R$-матрицы $R \in U_{q}\left(b_{+}\right) \otimes$ $U_{q}\left(b_{-}\right)$, представляя первую алгебру $U_{q}\left(b_{+}\right)$в квантовом пространстве, а вторую $U_{q}\left(b_{-}\right)$- с помощью матрищ со спектральным параметром (вспомогательное пространство). Подставляя генераторы $\Delta^{(N-1)} U_{q}\left(\widehat{s l}_{2}\right) \quad N$-й степени алгебры $U_{q}\left(\widehat{s l}_{2}\right)$ (взятые в качестве квантового представления) в формулу для универсальной $R$-матрицы, получим матрицу монодромии на $N$ узлах (8).

Проиллюстрируем вывод уравнения ЯБ (7) из универсального уравнения $(9)$, которое является уравнением в $U_{q}\left(\widehat{s l}_{2}\right) \otimes U_{q}\left(\widehat{s l}_{2}\right) \otimes U_{q}\left(\widehat{s l}_{2}\right)$. Чтобы получить уравнение $(7)$, представим первую алгебру в квантовом пространстве $h_{n}$, а вторую и третью - в конечномерном матричном представлении со спектральным параметром.

\section{2. ЯВНОЕ ВЫЧИСЛЕНИЕ $L$-ОПЕРАТОРА ВОЛЬТЕРРА ИЗ УНИВЕРСАЛЬНОЙ $R$-МАТРИЦЫ}

Напомним, что квантовый $L$-оператор модели Вольтерра

$$
L(\lambda)=\left(\begin{array}{cc}
u & -\lambda v^{-1} \\
\lambda v & u^{-1}
\end{array}\right)
$$

был введен в [3] при помощи пары Вейля $u$ и $v$,

$$
u v=q v u
$$

$L$-оператор удовлетворяет уравнению ЯБ (7) для тригонометрической $R$-матрищы. Для вывода $L$-оператора (13) из универсальной $R$-матрицы используем представление $q$-осцилляторов, важность которого была подчеркнута в [9]. Было показано, что для получения оператора Бакстера $Q(\lambda)$ из $R=\sum_{i} A_{i} \otimes B_{i} \in U_{q}\left(b_{+}\right) \otimes U_{q}\left(b_{-}\right)$нужно использовать представление $q$-осцилляторов $V_{ \pm}$для второй алгебры $U_{q}\left(b_{-}\right)$, когда

$$
e_{\alpha_{0}} e_{\alpha_{1}}-q^{2} e_{\alpha_{1}} e_{\alpha_{0}}=\frac{\lambda^{2}}{q^{-2}-1} \quad\left(\text { представление } V_{+}(\lambda)\right)
$$

и

$$
e_{\alpha_{1}} e_{\alpha_{0}}-q^{2} e_{\alpha_{0}} e_{\alpha_{1}}=\frac{\lambda^{2}}{q^{-2}-1} \quad\left(\text { представление } V_{-}(\lambda)\right)
$$


Имеем

$$
Q_{+}(\lambda)=\operatorname{tr}_{V_{+}(\lambda)} R, \quad Q_{-}(\lambda)=\operatorname{tr}_{V_{-}(\lambda)} R
$$

для любого квантового представления $U_{q}\left(b_{+}\right)$(для любой интегрируемой модели $)^{1}$. Такие $Q$-операторы удовлетворяют уравнению Бакстера (6).

Здесь представление $q$-осцилляторов (как вспомогательное) описывает сорт объектов КМОЗ: он дает $Q$-оператор Бакстера для любого квантового представления алгебры $U_{q}\left(b_{+}\right)$.

Возникает вопрос: какая интегрируемая модель соответствует представлению $q$-осцилляторов?

Покажем, что такой моделью является квантовая модель Вольтерра. Для конструкции ее $L$-оператора мы используем частный случай представления $V_{ \pm}(\lambda)$. Именно, определим представление $W(\lambda)$ алгебры $U_{q}\left(b_{+}\right)$следующим образом:

$$
e_{\alpha_{1}}=\lambda e\left(q-q^{-1}\right)^{-1}, \quad e_{\alpha_{0}}=\lambda e^{-1}\left(q-q^{-1}\right)^{-1}, \quad k_{\alpha_{1}}=k\left(=q^{h}\right),
$$

где $k e=q^{2} e k$. Представление $V_{ \pm}(\lambda)$ вырождается в коммутирующие генераторы $e_{\alpha_{1}}$ и $e_{\alpha_{0}}$.

Согласно определению генераторов нашей алгебры (10) имеем

$$
e_{\delta}^{\prime}=\frac{\lambda^{2}}{q^{2}-1}
$$

Используя преобразования (11), получим, что

$$
\begin{gathered}
e_{n \delta}=(-)^{n+1} \frac{q^{-n}}{q-q^{-1}} \frac{\lambda^{2 n}}{n}, \\
e_{\alpha_{1}+l \delta}=e_{\alpha_{0}+l \delta}=0 \quad \text { для } \quad l=1,2, \ldots .
\end{gathered}
$$

Чтобы получить $L$-оператор модели Вольтерра, рассмотрим представление операторов алгебры $U_{q}\left(\widehat{s l}_{2}\right)$ в терминах $U_{q}\left(s l_{2}\right) \otimes C\left[\lambda, \lambda^{-1}\right]$ :

$$
e_{ \pm \alpha_{1}}=\lambda^{ \pm 1} e_{ \pm \alpha}, \quad e_{ \pm \alpha_{0}}=-\lambda^{ \pm 1} e_{\mp \alpha}, \quad k_{\alpha_{1}}=k_{\alpha}
$$

где алгебра $U_{q}\left(s l_{2}\right)$ имеет генераторы $e_{ \pm \alpha}$ и $k_{\alpha}^{ \pm 1}$ с обычными коммутационными соотношениями

$$
k_{\alpha} e_{ \pm \alpha}=q^{ \pm 2} e_{ \pm \alpha} k_{\alpha}, \quad\left[e_{\alpha}, e_{-\alpha}\right]=\frac{k_{\alpha}-k_{\alpha}^{-1}}{q-q^{-1}}
$$

Введем неприводимое 2-мерное матричное представление алгебры $U_{q}\left(s l_{2}\right)$ :

$$
e_{\alpha}=\left(\begin{array}{cc}
0 & 1 \\
0 & 0
\end{array}\right), \quad e_{-\alpha}=\left(\begin{array}{cc}
0 & 0 \\
1 & 0
\end{array}\right), \quad k_{\alpha}=\left(\begin{array}{cc}
q & 0 \\
0 & q^{-1}
\end{array}\right) .
$$

\footnotetext{
1) Мы выбираем бесконечномерное пространство представлений $V_{ \pm}(\lambda)$ так, что след $Q_{ \pm}(\lambda)=$ $\operatorname{tr}_{V_{ \pm}(\lambda)} R$ сушествует.
} 
Теперь мы можем определить представление операторов аффинной алгебры $U_{q}\left(\widehat{s l}_{2}\right)$ из (18) и представление (19) для простой алгебры $U_{q}\left(s l_{2}\right)$ :

$$
e_{\alpha_{1}+m \delta}=\lambda^{2 m+1} k_{\alpha}^{-m} e_{\alpha}, \quad e_{\alpha_{0}+m \delta}=-\lambda^{2 m+1} e_{-\alpha} k_{\alpha}^{-m}
$$

и

$$
e_{-\left(\alpha_{1}+m \delta\right)}=\lambda^{-2 m-1} e_{-\alpha} k_{\alpha}^{m}, \quad e_{-\left(\alpha_{0}+m \delta\right)}=-\lambda^{-2 m-1} k_{\alpha}^{m} e_{\alpha}
$$

Для векторов, соответствующих мнимым корням, имеем

$$
\begin{gathered}
e_{m \delta}=-\frac{q^{m}+q^{-3 m}-k_{\alpha}^{-m}-q^{-2 m} k_{\alpha}^{-m}}{m\left(q-q^{-1}\right)} \lambda^{2 m} \\
e_{-m \delta}=\frac{q^{-m}+q^{3 m}-k_{\alpha}^{m}-q^{2 m} k_{\alpha}^{m}}{m\left(q-q^{-1}\right)} \lambda^{-2 m}
\end{gathered}
$$

Теперь все готово для вывода $L$-оператора модели Вольтерра из универсальной $R$-матрицы. Обозначим через $L_{1 / 2}(\lambda)$ некоторый $L$-оператор, полученный из $R=\sum_{i} A_{i} \otimes$ $B_{i} \in U_{q}\left(b_{+}\right) \otimes U_{q}\left(b_{-}\right)$с помощью такого представления, когда первая алгебра $U_{q}\left(b_{+}\right)$ представлена в квантовом пространстве $W(\lambda)$, а вторая алгебра $U_{q}\left(b_{-}\right)$- во вспомогательном пространстве спина $1 / 2$.

Первое и третье произведения в формуле (12) с учетом свойства (17) имеют вид

$$
\begin{gathered}
\left(\prod_{n \geq 0}^{\rightarrow} \exp _{q^{-2}}\left(\left(q-q^{-1}\right) e_{\alpha_{1}+n \delta} \otimes e_{-\alpha_{1}-n \delta}\right)\right)= \\
\quad=1+\left(q-q^{-1}\right) e_{\alpha_{1}} \otimes e_{-\alpha_{1}}=\left(\begin{array}{cc}
1 & 0 \\
\lambda e & 1
\end{array}\right) \\
\left(\prod_{n \geq 0}^{\leftarrow} \exp _{q^{-2}}\left(\left(q-q^{-1}\right) e_{\alpha_{0}+n \delta} \otimes e_{-\alpha_{0}-n \delta}\right)\right)= \\
\quad=1+\left(q-q^{-1}\right) e_{\alpha_{0}} \otimes e_{-\alpha_{0}}=\left(\begin{array}{cc}
1 & -\lambda e^{-1} \\
0 & 1
\end{array}\right)
\end{gathered}
$$

Используя формулы (16) и (23), для второго множителя в (12) (с точностью до константы) имеем

$$
\begin{aligned}
& \exp \left(\sum_{n>0}\left(q-q^{-1}\right) \frac{n\left(e_{n \delta} \otimes e_{-n \delta}\right)}{[2 n]_{q}}\right)= \\
& \quad=\exp \left(\left(q-q^{-1}\right) \sum_{n>0}(-)^{n} \frac{\lambda^{2 n}}{n}\right)\left(\begin{array}{ll}
1 & 0 \\
0 & 0
\end{array}\right)=\left(\begin{array}{cc}
\frac{1}{1+\lambda^{2}} & 0 \\
0 & 1
\end{array}\right) .
\end{aligned}
$$

Множитель $\mathcal{K}$ сводится к выражению

$$
\mathcal{K}=q^{\frac{h_{\alpha} \otimes h_{\alpha}}{2}}=\left(\begin{array}{cc}
k^{\frac{1}{2}} & 0 \\
0 & k^{-\frac{1}{2}}
\end{array}\right) .
$$


Таким образом, оператор $L_{1 / 2}(\lambda)$ принимает вид

$$
L_{\frac{1}{2}}(\lambda)=\left(\begin{array}{cc}
k^{\frac{1}{2}} & -\lambda e^{-1} k^{-\frac{1}{2}} \\
\lambda e k^{\frac{1}{2}} & k^{-\frac{1}{2}}
\end{array}\right) .
$$

Выбирая $e=v u^{-1}$ и $k=u^{2}$, мы получаем, что $L_{1 / 2}(\lambda)$ совпадает с $L$-оператором модели Вольтерра (13).

\section{3. ФУНДАМЕНТАЛЬНАЯ $R$-МАТРИЦА МОДЕЛИ ВОЛЬТЕРРА}

В этом разделе мы построим фундаментальную $R$-матрицу модели Вольтерра $R_{f}$ $[3,13]$. Введем представление $U_{q}\left(b_{-}\right)$алгебры $\widetilde{W}(\lambda)$ :

$$
e_{-\alpha_{1}}=\lambda^{-1} \tilde{e}^{-1}\left(q-q^{-1}\right)^{-1}, \quad e_{-\alpha_{0}}=\lambda^{-1} \tilde{e}\left(q-q^{-1}\right)^{-1}, \quad k_{\alpha_{1}}=\tilde{k}\left(=q^{\tilde{h}}\right),
$$

где $\tilde{k} \tilde{e}=q^{2} \tilde{e} \tilde{k}$, причем остальные генераторы подалгебры $U_{q}\left(b_{-}\right)$имеют вид

$$
e_{-\alpha_{1}+l \delta}=e_{-\alpha_{0}+l \delta}=0, \quad e_{-(l+1) \delta}=\mathrm{const} \quad \text { для } \quad l=1,2, \ldots
$$

Чтобы получить $R_{f}$ из $R=\sum_{i} A_{i} \otimes B_{i} \in U_{q}\left(b_{+}\right) \otimes U_{q}\left(b_{-}\right)$, необходимо представить алгебру $U_{q}\left(b_{+}\right)$в квантовом пространстве $W(\lambda)(15)-(17)$, а алгебру $U_{q}\left(b_{-}\right)-$в пространстве $\widetilde{W}(\lambda)(25),(26)$. Согласно определению фундаментальной $R$-матрицы ее квантовое пространство совпадает со вспомогательным.

Таким образом, второй и третий множители в формуле (12) имеют вид

$$
\begin{aligned}
& \left(\prod_{n \geq 0}^{\rightarrow} \exp _{q^{-2}}\left(\left(q-q^{-1}\right) e_{\alpha_{1}+n \delta} \otimes e_{-\alpha_{1}-n \delta}\right)\right)=\exp _{q^{-2}}\left(\left(q-q^{-1}\right)^{-1} e \otimes \tilde{e}^{-1} \lambda\right) \\
& \left(\prod_{n \geq 0}^{\leftarrow} \exp _{q^{-2}}\left(\left(q-q^{-1}\right) e_{\alpha_{0}+n \delta} \otimes e_{-\alpha_{0}-n \delta}\right)\right)=\exp _{q^{-2}}\left(\left(q-q^{-1}\right)^{-1} e^{-1} \otimes \tilde{e} \lambda\right)
\end{aligned}
$$

в соответствии с формулами (17) и (26). Далее мы видим, что мнимые корни для $U_{q}\left(b_{+}\right)$ и $U_{q}\left(b_{-}\right)$являются константами. Следовательно, мы можем опустить второй множитель, содержаший экспоненту от мнимых корней.

Имеем

$$
R_{f}(\lambda)=\exp _{q^{-2}}\left(\left(q-q^{-1}\right)^{-1} e \otimes \tilde{e}^{-1} \lambda\right) \exp _{q^{-2}}\left(\left(q-q^{-1}\right)^{-1} e^{-1} \otimes \tilde{e} \lambda\right) q^{\frac{h \otimes \tilde{h}}{2}}
$$

Покажем место фундаментальной $R$-матрицы в уравнении ЯБ. Универсальная форма уравнения ЯБ имеет вид ${ }^{2)}$

$$
R_{12} R_{13} R_{23}=R_{23} R_{13} R_{12} \in U_{q}\left(b_{+}\right) \otimes U_{q}\left(\widehat{s l}_{2}\right) \otimes U_{q}\left(b_{-}\right)
$$

\footnotetext{
2) Заметим, что продолжить представление $W(\lambda)$ с $U_{q}\left(b_{+}\right)$на целую алгебру $U_{q}\left(\widehat{s l_{2}}\right)$ невозможно.
} 
Здесь для первой алгебры $U_{q}\left(b_{+}\right)$мы выбираем представление $W(\lambda) \equiv n_{1}$, для второй алгебры $U_{q}\left(\widehat{s l}_{2}\right)$ - представление, тождественно равное $a$, и для третьей - представление $\widetilde{W}(\lambda) \equiv n_{2}$.

Таким образом, имеем

$$
L_{n_{1}, a}(\lambda) R_{n_{1}, n_{2}}(\mu \lambda) L_{a, n_{2}}(\mu)=L_{a, n_{2}}(\mu) R_{n_{1}, n_{2}}(\mu \lambda) L_{n_{1}, a}(\lambda)
$$

где $L_{n_{1}, a}(\lambda)-L$-оператор модели Вольтерра и $R_{n_{1}, n_{2}}(\lambda) \equiv R_{f}(\lambda)$ - фундаментальная $R$-матрица модели Вольтерра.

Обозначим след матрицы монодромии для фундаментальной $R$-матрицы следующим образом:

$$
I(\lambda)=\operatorname{tr}_{h}\left[R_{N, h}(\lambda) R_{N-1, h}(\lambda) \ldots R_{1, h}(\lambda)\right] .
$$

Уравнение (28) гарантирует коммутативность $I(\lambda)$ и следа $T_{a}(\lambda)=\operatorname{tr}_{\nu_{a}} M_{a}(\lambda)$.

Записав генераторы в терминах пары Вейля (14):

$$
\begin{array}{ll}
e=v u^{-1}, & k=u^{2}, \\
\tilde{e}=v u^{-3}, & \tilde{k}=u^{2},
\end{array}
$$

получаем для $R_{f}(\lambda)$ выражение

$$
R_{f}(\lambda)=\exp _{q^{-2}}\left(\left(q-q^{-1}\right)^{-1} v u^{-1} \otimes u^{3} v^{-1} \lambda\right) \exp _{q^{-2}}\left(\left(q-q^{-1}\right)^{-1} u v^{-1} \otimes v u^{-3} \lambda\right) q^{\frac{h_{\alpha} \otimes h_{\alpha}}{2}}
$$

После нормализации находим формулу для фундаментальной $R$-матрицы

$$
r(\lambda, w)=R_{f}(1)^{-1} R_{f}(\lambda)=\frac{\exp _{q^{-2}}\left(\left(q-q^{-1}\right)^{-1} w \lambda\right) \exp _{q^{-2}}\left(\left(q-q^{-1}\right)^{-1} w^{-1} \lambda\right)}{\exp _{q^{-2}}\left(\left(q-q^{-1}\right)^{-1} w\right) \exp _{q^{-2}}\left(\left(q-q^{-1}\right)^{-1} w^{-1}\right)}
$$

где $w=v u \otimes u v^{-1}$.

Действительно, функция $r(\lambda, w)$ удовлетворяет уравнению ЯБ

$$
r\left(\lambda, w_{12}\right) r\left(\lambda \mu, w_{23}\right) r\left(\mu, w_{12}\right)=r\left(\mu, w_{23}\right) r\left(\lambda \mu, w_{12}\right) r\left(\lambda, w_{23}\right)
$$

с $w_{12}=v u \otimes u v^{-1} \otimes 1$ и $w_{23}=1 \otimes v u \otimes u v^{-1}$. Решение этого уравнения было найдено в [13] и имеет вид

$$
r(\lambda, w)=1+\sum_{k=1}^{\infty} \frac{(1-\lambda)\left(q-\lambda q^{-1}\right) \ldots\left(q^{k-1}-\lambda q^{-k+1}\right)}{\left(q^{-1}-\lambda q\right) \ldots\left(q^{-k}-\lambda q^{k}\right)} w^{k}
$$

В [3] было замечено, что $r$-матрица $r(\lambda, w)$ удовлетворяет функциональному уравнению

$$
\frac{r(\lambda, w q)}{r\left(\lambda, w q^{-1}\right)}=\frac{1+\lambda w}{\lambda+w}
$$


Для доказательства этого факта достаточно представить $q$-экспоненту как бесконечное произведение

$$
\exp _{q^{-2}}\left(\left(q-q^{-1}\right)^{-1} w\right)=\prod_{k=0}^{\infty}\left(1+q^{2 k+1} w\right)
$$

Мультипликативная форма (29) была найдена в [14].

Можно написать стандартную форму уравнения ЯБ для фундаментальной $R$-матрицы $\operatorname{Pr}(\lambda)(29)$ и $L$-оператора Вольтерра $(13)^{3)}$ :

$$
L_{n_{2}, a}(\lambda) L_{n_{1}, a}(\mu) \operatorname{Pr}(\mu / \lambda)=\operatorname{Pr}(\mu / \lambda) L_{n_{1}, a}(\mu) L_{n_{2}, a}(\lambda)
$$

\section{4. ОБСУЖДЕНИЕ И ЗАКЛЮЧЕНИЕ}

В этой работе была показана роль универсальной $R$-матрицы в формализме КМОЗ на примере квантовой модели Вольтерра.

На основе универсальной $R$-матрицы $R=\sum_{i} A_{i} \otimes B_{i} \in U_{q}\left(b_{+}\right) \otimes U_{q}\left(b_{-}\right)$установлено, что квантовое представление модели Вольтерра совпадает с представлением $q$-осцилляторов. Таким образом, мы вывели $L$-оператор Вольтерра и фундаментальную $R$-матрицу. Вырожденное представление $q$-осцилляторов является простейшим для алгебры $U_{q}\left(b_{+}\right)$.

Было бы интересно обобщить взаимнооднозначное соответствие между интегрируемой системой и квантовым представлением универсальной $R$-матришы $q$-осцилляторов на другие представления $U_{q}\left(b_{+}\right)$.

Например, пусть $V_{\mu}$ - модуль Верма для $U_{q}\left(s l_{2}\right)$ со старшим весом $\mu \in C$. Тогда обозначим представление вычисления аффинной алгебры $U_{q}\left(\widehat{s l}_{2}\right)$ как $V_{\mu}(\lambda)$. Квантовый $L$-оператор, полученный из универсальной $R$-матрицы имеет вид

$$
L(\lambda)=\left(\begin{array}{cc}
k_{\alpha}^{\frac{1}{2}}-\lambda^{2} q^{-1} k_{\alpha}^{-\frac{1}{2}} & -\lambda e_{-\alpha} k_{\alpha}^{-\frac{1}{2}}\left(q-q^{-1}\right) \\
\lambda e_{\alpha} k_{\alpha}^{\frac{1}{2}}\left(q-q^{-1}\right) & k_{\alpha}^{-\frac{1}{2}}-\lambda^{2} q^{-1} k_{\alpha}^{\frac{1}{2}}
\end{array}\right),
$$

где $e_{ \pm \alpha}$ и $k_{\alpha}^{ \pm 1}$ - генераторы алгебры $U_{q}\left(s l_{2}\right)$. Этот $L$-оператор напоминает $L$-оператор для решеточной модели синус-Гордон [15].

\footnotetext{
3) $P$ - оператор перестановки: $P\left(w_{1} \otimes w_{2}\right)=w_{2} \otimes w_{1}$.
} 


\section{Список литературы}

[1] В.Е. Захаров, С.В. Манаков, С.П. Новиков, Л.П. Питаевский. Теория солитонов. Метод обратной задачи. М.: Наука, 1980.

[2] L. D. Faddeev, L. A. Takhtajan. Springer Lecture Notes in Physics. 1986. V. 246. P. 166.

[3] A. Volkov. Phys. Lett. A. 1992. V. 167. P. 345.

[4] O. Babelon. Phys. Lett. B. 1990. V. 238. P. 234.

[5] Е.К. Склянин, Л. А. Тахтаджян, Л. Д. Фаддеев. ТМФ. 1979. Т. 40. С. 194.

[6] S. Khoroshkin, A. Stolin. V. Tolstoy. Mod. Phys. Lett. A. 1995. V. 10. № 19. P. 1375.

[7] L. Faddeev. Int. J. Mod. Phys. A. 1995. V. 10. № 13. P. 1845.

[8] R. Baxter. Exactly Solved Models in Statistical Mechanics. London: Academic Press, 1982.

[9] V. Bazhanov, S. Lukyanov, A. Zamolodchikov. Integrable Structure of Conformal Field Theory II. $Q$-operator and DDV equation. hep-th/9604044.

[10] E. Damaskinsky, P. Kulish. Irreducible representations of deformed oscillator algebra and $q$-special functions. q-alg/9610002.

[11] A. Antonov, B. Feigin. Phys. Lett. B. 1997. V. 392. P. 115.

[12] В. О. Тарасов, Л. А. Тахтаджсян, Л. Д. Фаддеев. ТМФ. 1983. Т. 57. С. 163.

[13] V. Fateev, A. B. Zamolodchikov. Phys. Lett. A. 1982. V. 92. P. 37.

[14] L. D. Faddeev, A. Volkov. Phys. Lett. B. 1993. V. 315. P. 311.

[15] A. Izergin, V. Korepin. Lett. Math. Phys. 1981. V. 5. P. 199.

Поступила в редакцию 24.IV.1997 г.

\section{A. V. Antonov \\ QUANTUM VOLTERRA MODEL AND UNIVERSAL $R$-MATRIX}

In this paper, we explicitly prove that an integrable system solved by the quantum inverse scattering method can be described by a pure algebraic object (universal $R$-matrix) and a proper algebraic representation. For the example of the quantum Volterra model, we construct the $L$-operator and the fundamental $R$-matrix from the universal $R$-matrix for the quantum affine $U_{q}\left(\widehat{s l}_{2}\right)$ algebra and $q$-oscillator representation for it. In this way, there is an equivalence between the integrable system with the symmetry algebra $\mathcal{A}$ and the representation of this algebra. 